\title{
Mexique : tensions et utopies autour de la réforme dans les États d'Oaxaca et du Chiapas
}

\section{Norma Georgina Gutiérrez}

Traducteur : Philippe Rabaté

\section{OpenEdition Journals}

Édition électronique

URL : https://journals.openedition.org/ries/5576

DOI : 10.4000/ries.5576

ISSN : 2261-4265

Éditeur

France Education international

Édition imprimée

Date de publication : 1 décembre 2016

Pagination : 15-19

ISBN : 978-2-85420-612-8

ISSN : $1254-4590$

\section{Référence électronique}

Norma Georgina Gutiérrez, « Mexique : tensions et utopies autour de la réforme dans les États d'Oaxaca et du Chiapas », Revue internationale d'éducation de Sèvres [En ligne], 73 | décembre 2016, mis en ligne le 01 décembre 2018, consulté le 01 juillet 2021. URL : http://journals.openedition.org/ries/ 5576 ; DOI : https://doi.org/10.4000/ries.5576

Ce document a été généré automatiquement le 1 juillet 2021

(c) Tous droits réservés 


\title{
Mexique : tensions et utopies autour de la réforme dans les États d'Oaxaca et du Chiapas
}

\author{
Norma Georgina Gutiérrez
}

Traduction : Philippe Rabaté

1 Depuis 1910, la constitution du Mexique stipule que l'éducation est obligatoire, laïque et gratuite (article 3). Le 20 décembre 2012, le Sénat de la République a approuvé une réforme éducative qui comportait de nombreuses modifications de cet article, et introduisait notamment la notion de qualité éducative. Les principaux changements portaient sur :

- l'évaluation des élèves de manière centralisée et soumise à un contrôle extérieur aux établissements ;

- l'entrée des enseignants dans le système éducatif sur concours ouvert à des candidats n'ayant pas nécessairement reçu de formation préalable et pouvant être issus de n'importe quel cursus universitaire ;

- l'évaluation électronique (examen) pour l'affectation et la titularisation des enseignants; cette évaluation a requis l'habilitation de milliers d'évaluateurs qui pouvaient ne pas être professeurs et n'avoir même aucune formation en éducation. C'est ainsi que s'est mis en place un système d'évaluation qui prétend s'appliquer à 1,6 million d'enseignants et qui a débuté avec 5000 évaluateurs " certifiés », au terme d'une formation de seulement quelques heures.

2 L'évaluation massive des élèves, des candidats qui souhaitent obtenir un poste et être titularisés ainsi que celle des enseignants déjà en exercice a constitué le cœur de ce que l'on a appelé la réforme éducative, réforme qui, jusqu'en août 2016, soit plus de trois ans après avoir été votée, ne contenait pas la moindre approche pédagogique, aucune proposition ou aucun nouveau plan d'études. Cette évaluation, qui n'a débuté qu'en 2015 et 2016, a entraîné la suspension ou le renvoi de milliers d'enseignants. Les enseignants sont soumis à un nouveau type de contrat dans lequel ils perdent leurs droits traditionnels et sont assujettis à de nouvelles conditions de travail, à une 
évaluation tous les quatre ans, qui conditionne la possibilité de continuer à enseigner ou l'exclusion du corps des enseignants.

3 L'Institut national d'évaluation éducative (INEE) est chargé de l'évaluation des enseignants ; cet organisme n'appartient pas à la structure du système d'éducation de base - où travaillent des chercheurs mexicains confirmés en éducation. L'INEE a sollicité, moyennant salaire, des institutions et des chercheurs en éducation afin qu'ils participent à ce processus, mais n'a pas jugé opportun d'y faire participer les représentants syndicaux des enseignants de l'éducation de base. Si l'on en juge d'après les actions, les outils, les applications développées, le nombre d'évaluateurs individuels et institutionnels impliqués, on peut imaginer l'investissement considérable que suppose la mise en place d'un tel système.

4 Interlocuteur privilégié de la direction de l'INEE, l'organisation Mexicanos Primero, financée par Televisa, la télévision la plus puissante du pays et de toute l'Amérique latine, est un autre acteur décisif de cette réforme. Cette chaîne a en effet utilisé une partie de son temps d'antenne - pourtant fort coûteux - pour présenter les enseignants mobilisés contre la réforme comme des vandales, des irresponsables ou des personnes faibles et elle a orchestré une véritable campagne de décrédibilisation et de criminalisation de leur mouvement, campagne qui alimente également le racisme et les agressions contre les enseignants indigènes et leurs communautés (Pérez Rocha, 2016).

C'est dans ce contexte d'évaluation nationale de l'éducation qu'est apparue une autre série d'actions à l'initiative du pouvoir : mise en place de droits d'inscription pour les élèves de l'éducation de base, refus du gouvernement de continuer à payer les services d'eau et de lumière des établissements, ainsi que l'entretien des bâtiments, appel à l'élaboration de programmes de formation d'enseignants en ligne qui ne dépendraient d'aucune école de formation des maitres, habilitation d'enseignants par des universités privées mexicaines, alors que, depuis des années, les écoles normales du pays, et tout particulièrement celles des zones rurales, qui accueillent des populations indigènes, se trouvent dans une situation d'abandon financier.

6 Les enseignants ont exprimé durant trois ans leur rejet de cette politique fédérale et, à partir de juin 2016, ils ont mené des mobilisations partielles, des arrêts de travail échelonnés et des grèves générales dans certains États mexicains. À ce climat de mécontentement croissant se sont ajoutées les actions de protestation menées à la suite du massacre commis à Iguala (État de Guerrero) qui a frappé 43 élèves de l'école normale rurale d'Ayotzinapa, en 2014.

7 Pour les enseignants de l'éducation de base, la réforme éducative actuelle abandonne le caractère généraliste et humaniste de l'éducation, met en danger sa gratuité et signifie un affaiblissement des droits des travailleurs. Ils se sont donc prononcés en faveur de l'abrogation de la réforme. En 2016, des grèves illimitées d'enseignants ont débuté dans les États de Michoacán, d'Oaxaca, de Guerrero et du Chiapas, autrement dit les plus pauvres, grâce à la Coordination nationale des travailleurs de l'éducation (CNTE). Il a fallu attendre le massacre perpétré par la police fédérale le 19 juin 2016 à Nochixtlán pour que l'État mexicain accepte de reconnaître la CNTE comme interlocuteur. Au cours de ces mois de mobilisation, le gouvernement a d'abord tenté le passage en force, avant de recourir à une stratégie d'usure du mouvement par des négociations menées parallèlement à une répression plus sélective. 


\section{Ce que signifie la réforme éducative à Nochixtlán}

8 La ville de Nochixtlán, dans l'État d'Oaxaca, compte actuellement environ 13000 habitants. Ce site de peuplement préhispanique, d'origine mixtèque, peut être considéré comme un exemple de survivance indigène, après plus de 500 ans d'exploitation et de traitement discriminatoire et, plus récemment et jusqu'à nos jours, par les assauts violents du narcotrafic. La population d'Oaxaca s'élève à environ 4 millions d'habitants. C'est l'État qui compte le plus de locuteurs de langue indigène au Mexique : mixtèque, zapotèque, mazatèque et mixe (INEGI, 2010).

9 Historiquement, Oaxaca et Chiapas ont les indices les plus élevés de pauvreté et les rangs les plus bas d'investissement fédéral pour les programmes économiques et productifs. Le programme fédéral «escuelas al 100 » (« des écoles à $100 \%$ ») reconnaît qu'il existe, dans cet état comme au Chiapas, des écoles sans toilettes ni électricité.

10 Le 19 juin à Nochixtlán, la police fédérale a tiré sur la population qui avait bloqué la route donnant accès à la ville pour soutenir les enseignants. Onze personnes sont mortes et des centaines de personnes ont été arrêtées, blessées ou ont disparu. Le gouverneur de l'État a invoqué la nécessité de sauvegarder les libertés, l'état de droit et l'intégrité physique et patrimoniale d'Oaxaca. La population indigène de la ville a immédiatement reçu des témoignages de solidarité des autres peuples de la région triquis, zapotèques, mixes, mazatèques et chatinos - qui ont repris à leur compte la demande d'abrogation de la réforme éducative (Hernández Navarro, 2016). La société civile a alors réclamé que soit établi un dialogue avec les enseignants et la fin de la répression. Les enseignants de Michoacán, du Chiapas et d'Oaxaca sont soutenus par les citoyens et, à partir du mois de juillet, des écoles de la ville de Mexico rejoignent le mouvement de grève.

\section{La construction d'un projet éducatif démocratique et l'expérience des peuples indigènes}

11 Outre la forte mobilisation pour exiger l'abrogation de la réforme, la CNTE a promu le dialogue et la négociation pour la remise en liberté des détenus, la justice pour les populations indigènes de Noxchitlán et pour la construction d'un projet éducatif démocratique. Des chercheurs en éducation et des intellectuels mexicains contribuent également à ce projet.

De son côté, le gouvernement a dû reconnaître les erreurs techniques et politiques de l'évaluation déjà mise en place et instaurer une étape de "transition ", à partir d'août 2016. Il a également remis en liberté des enseignants qui avaient été arrêtés à Oaxaca et modifié en partie les coupes salariales déjà mises en œuvre à la suite de la mise en place des nouvelles formes d'évaluation. Toutefois, l'État ne répond pas à la demande d'abrogation de la réforme. Les enseignants de la CNTE exigent de pouvoir participer directement à la construction du projet éducatif et appellent les parents, les élèves, les communautés et la société civile à y prendre part. Ils s'opposent au modèle officiel rendu public en août 2016, tant celui-ci est uniforme, national, encyclopédique et étranger à la diversité des réalités du pays. Ils lui reprochent également d'avoir été élaboré sans eux. 
13 La lutte des enseignants a donné lieu à de nombreux débats, réunions, commissions, forums d'enseignants et d'universitaires, où se sont exprimées des idées importantes en matière d'éducation de base :

- l'éducation de base est l'affaire et la mission de professionnels expressément formés pour cette tâche ;

- l'école normale est réaffirmée comme étant la voie de formation pour s'occuper des écoles d'éducation de base ;

- un appel général est lancé pour la construction de projets éducatifs à partir du travail quotidien des enseignants dans les salles de classe, avec la participation des élèves, des parents et de la communauté ;

- on reconnaît et on exige l'appui des universitaires et chercheurs pour mener la réflexion sur cette tâche ;

- ce questionnement est étendu aussi bien aux modèles éducatifs mis en œuvre dans les classes qu'à ceux qui sont imposés d'en haut ;

- les peuples indigènes possèdent leurs propres constructions de projets éducatifs et celles-ci sont anciennes.

14 Un regard plus circonstancié sur les projets éducatifs des peuples indigènes permet d'identifier des caractéristiques communes. Avant tout, les expériences liées à ces projets révèlent un sens du bien commun par rapport à l'éducation, à la manière de vivre l'école dans et avec la communauté. On y fait référence à de longs processus de construction de projets éducatifs communautaires, qui réussissent grâce à des actions collectives et qui permettent de définir des contenus pour l'éducation de base mais également de coproduire des connaissances adaptées aux contextes et aux besoins des communautés scolaires et des populations. L'une des plus importantes organisations collectives est l'assemblée communautaire, qui s'intéresse avant tout aux biens collectifs et participe directement à l'établissement des contenus enseignés à l'école. Nous en citons ici quelques exemples.

Le respect et la protection de l'environnement de l'école, par ceux qui y vivent, consiste concrètement à éduquer aux valeurs de défense de la terre, des forêts, de l'eau, de la flore, la faune et des ressources minérales. L'apprentissage de la culture et de la langue est intimement lié à la reconnaissance et à la conscience de se savoir possesseur d'une diversité technique et linguistique mais également géologique, archéologique, naturelle et alimentaire. C'est ici que figurent des enseignements sur la géographie et l'histoire, cette dernière se référant clairement aux générations passées.

En ce qui concerne la santé, les contenus enseignés font référence aux savoirs spécifiques, comme le droit à préserver et diffuser les connaissances sur les plantes médicinales et à enseigner leur culture et leur usage. Il s'agit de prendre en compte, à partir de leur usage dans la vie quotidienne, des savoirs, pratiques et connaissances qui ont nourri l'existence de ces communautés pendant des siècles.

La formation aux droits de l'homme, qui englobent aussi bien les droits citoyens que ceux des peuples autochtones, constitue un autre volet de contenus enseignés. Y figurent notamment la formation aux us et coutumes des peuples indigènes, la référence à la lutte pour conserver la terre et ses ressources, la défense et la revendication de la remise en liberté des détenus indigènes et des défenseurs de la terre, et la nécessité que soit rendue justice aux milliers d'indigènes disparus et assassinés. 
lieu, le respect de la parole donnée comme forme d'engagement collectif - afin de développer une éducation fondée sur la paix et la justice. Dans ce projet figure également la nécessité de transformer le lieu où l'on vit dans l'intérêt des élèves, des parents, des paysans et des populations de manière générale.

19 L'évaluation est considérée à Oaxaca comme un processus devant concerner un projet collectif et non un individu isolé, afin de prendre en compte le travail d'une personne dans sa communauté. Ceci vaut pour l'évaluation des étudiants et pour celle des enseignants. L'auto-évaluation joue un rôle central, et conduit nécessairement le maître et l'élève à réfléchir sur leur pratique et non sur leur relation à un modèle auquel se conformer, ce dernier étant du reste défini à la marge et contre les besoins des communautés. Pour les enseignants d'Oaxaca, l'évaluation donne lieu à une reconnaissance et non à une punition ou à une séparation des membres de la communauté scolaire. C'est une pratique de valorisation de l'action collective et non du travail isolé d'un individu. Dans ce processus, la participation des enseignants, des étudiants, des parents et de la communauté en général est conçue comme une création d'espaces pour la réflexion et le dialogue.

Cette approche éducative repose également sur un profil d'enseignant-type capable de réfléchir, d'analyser et d'approfondir, par exemple sur la manière de faire progresser le projet éducatif au sein d'une classe où il s'occupe simultanément d'élèves de différents niveaux.

21 Ces projets se proposent de former un citoyen respectueux des formes collectives d'organisation, d'une formation critique et d'une attitude qui favorise le changement, qu'il s'agisse de pratiques productives, de rassemblements politiques, de célébrations communautaires, de rituels et fêtes religieuses et, bien sûr, d'éducation.

\section{BIBLIOGRAPHIE}

HERNANDEZ NAVARRO L. (2016) : « La masacre de Nochxtitlán y la reforma educativa », La Jornada, 5 juillet [en ligne] [https://goo.gl/cLzR4p]

Consejo Nacional de Evaluación de la Política de Desarrollo Social CONEVAL (2014) : Informe de pobreza en Mexico 2012, Mexico : CONEVAL, 125 p. [en ligne] [https://goo.gl/izzjED]

Instituto Nacional de Estadística y Goegrafía (INEGI) (2010) : [https://goo.gl/8muzME]

PEREZ ROCHA M. (2016) : « La letra y el capital con sangre entran », La Jornada, 27 juin. [en ligne] [https://goo.gl/ZmdohW] 
INDEX

Mots-clés : politique éducative, réforme de l'enseignement, évaluation de l'enseignant

Palabras claves : política educacional, reforma de la educación, evaluación del docente

Keywords : educational policy, educational reform, teacher evaluation

Index géographique : Mexique

\section{AUTEURS}

\section{NORMA GEORGINA GUTIÉRREZ}

Norma Georgina Gutiérrez est chercheuse en éducation à l'Université nationale autonome de Mexico, où elle a obtenu une licence de psychologie. Titulaire d'un doctorat en éducation du Centre de recherches et d'études approfondies de l'Institut national polytechnique (Mexique), elle s'intéresse en particulier aux communautés et aux réseaux de recherche, à la culture, aux savoirs et à l'apprentissage. Courriel : georgtz@correo.crim.unam.mx 